\title{
Ribosyldihydronicotinamide Dehydrogenase [Quinone]
}

National Cancer Institute

\section{Source}

National Cancer Institute. Ribosyldihydronicotinamide Dehydrogenase [Quinone]. NCI

Thesaurus. Code C103986.

Ribosyldihydronicotinamide dehydrogenase [quinone] (231 aa, $\sim 26 \mathrm{kDa}$ ) is encoded by the human NQO2 gene. This protein is involved in the metabolism of quinone-containing compounds. 